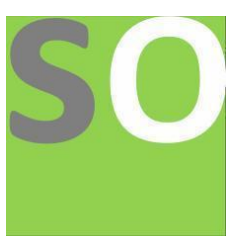

Article title: Adoption of machine learning for medical diagnosis

Authors: Mohammed Yousef Shaheen[1]

Affiliations: Saudia Arabia[1]

Orcid ids: 0000-0002-2993-2632[1]

Contact e-mail: yiroyo1235@tmednews.com

License information: This work has been published open access under Creative Commons Attribution License http://creativecommons.org/licenses/by/4.0/, which permits unrestricted use, distribution, and reproduction in any medium, provided the original work is properly cited. Conditions, terms of use and publishing policy can be found at https://www.scienceopen.com/.

Preprint statement: This article is a preprint and has not been peer-reviewed, under consideration and submitted to ScienceOpen Preprints for open peer review.

DOI: 10.14293/S2199-1006.1.SOR-.PPHMKA6.v1

Preprint first posted online: 25 September 2021

Keywords: ANN, Artificial Intelligence, CNN, Healthcare, Machine learning, Diagnosis, AI 


\title{
Adoption of machine learning for medical diagnosis
}

\author{
Mohammed Yousef Shaheen
}

The healthcare industry has historically been an early adopter of technology advancements and has reaped significant benefits. Machine learning (an artificial intelligence subset) is being used in a variety of health-related fields, including the invention of new medical treatments, the management of patient data and records, and the treatment of chronic diseases. One of the most important uses of machine learning in healthcare is the detection and diagnosis of diseases and conditions that are otherwise difficult to identify. This can range from tumors that are difficult to detect in their early stages to other hereditary illnesses. This research identifies and discusses the various usages of machine learning in medical diagnosis.

Keywords: ANN, Artificial Intelligence, CNN, Healthcare, Machine learning, Diagnosis,

\section{Introduction}

A late diagnosis of a disease, which results in delayed treatment and recovery, is fairly prevalent. A large number of people could be saved if we could diagnose an illness before it manifested in a person's body. Machine Learning technique is presently being investigated and used to speed up the diagnosis of numerous diseases, including cancer.

Machine learning is an Artificial Intelligence application that employs algorithms to discover patterns in massive volumes of data. Data can take many forms, including images, statistics, and words. This data is parsed by machine learning software, which then "learns" from it by drawing patterns from which it may generate predictions. With the goal of applying the learning to similar elements, the Machine Learning algorithm seeks for a system of rules that allow it to determine the general features of components within a group (Lee, 2021). 
Machine learning has gained popularity in healthcare due to its capacity to aid in the early detection of diseases in a reasonably speedy and accurate manner. With the medical field, machine learning can be utilized to aid in disease diagnosis. It's commonly utilized to aid in the detection of breast cancer utilizing ultrasound or X-ray imaging. The supervised learning classification is used in this circumstance since we want to know if there is cancer prevalent or not (a discrete binary label) (Saxena, 2021). As an added bonus, the algorithm can explain why it categorised an image the way it did, providing useful information to health professionals.

Another well-known example application of machine learning in healthcare is the prediction of Alzheimer's disease (Tanveer et al., 2020). The machine learning model in this example searches for patterns in the speech of participants with this ailment using a set of audio recordings. The amplitude and frequency of sounds, as well as the pauses between sentences, are used in the analysis. The model aids a geriatric medicine expert in detecting early Alzheimer's signs through speech.

Machine learning algorithms has the ability to work with a large amount of data. They do it as part of their search for essential linkages in the decision-making process. In the field of medicine, there is a vast amount of information to be detailed. People accustomed to preserve and archive this information, but modern technology has advanced. ML algorithms are set to deliver multidimensional solutions based on all obtained data (Panesar, 2019). Medical machine learning can also be utilized to find new explanations. Medical facilities can make better use of the information gathered (Shaheen, 2021a). Algorithms come very handy in this situation. The key to saving important doctors' time is effective information management. Physicians find it simpler to assist their patients with their ailments. Meanwhile, medical facilities can reduce the costs connected with various treatment procedures (Hu et al., 2015).

\section{Usage of Artificial Neural Network (ANN) and Deep ANN in medical diagnosis}

\subsection{ANN and DANN in medical diagnosis}

The artificial neural network (ANN) is a modelling approach motivated by the human nervous system that permits learning by pattern from representative data that depicts a physical occurrence or a decision process. The ability to build actual correlations between independent and dependent variables, as well as extract delicate information and complicated knowledge from representative data sets, is a distinguishing feature of ANN (Srivastav, 2021) (Kollár, 2021a). Without making any assumptions regarding a formal 
representation of the events, the connections between independent and dependent variables can be established. ANN models have several advantages over regression-based models, such as the ability to handle noisy data (Sadiq et al., 2019).

A layer of input nodes and a layer of output nodes make up an ANN, which is linked by one or more layers of hidden nodes. By firing activation functions, input layer nodes send information to hidden layer nodes, while hidden layer nodes either fire or stay latent depending on the evidence given (Kollár, 2021b). The evidence is weighted by the hidden layers, and when the value of a node or collection of nodes in the hidden layer hits a certain threshold, a value is passed to one or more nodes in the output layer. A vast number of cases must be used to train ANNs (data). For infrequent or extreme events, where data is inadequate to train the model, ANNs cannot be used. Human expertise cannot be used as a replacement for quantitative evidence in ANNs (Sadiq et al., 2019).

A deep learning algorithm is a neural network that has more than three layers, including the inputs and outputs. A two- or three-layer neural network is referred to as a simple neural network.

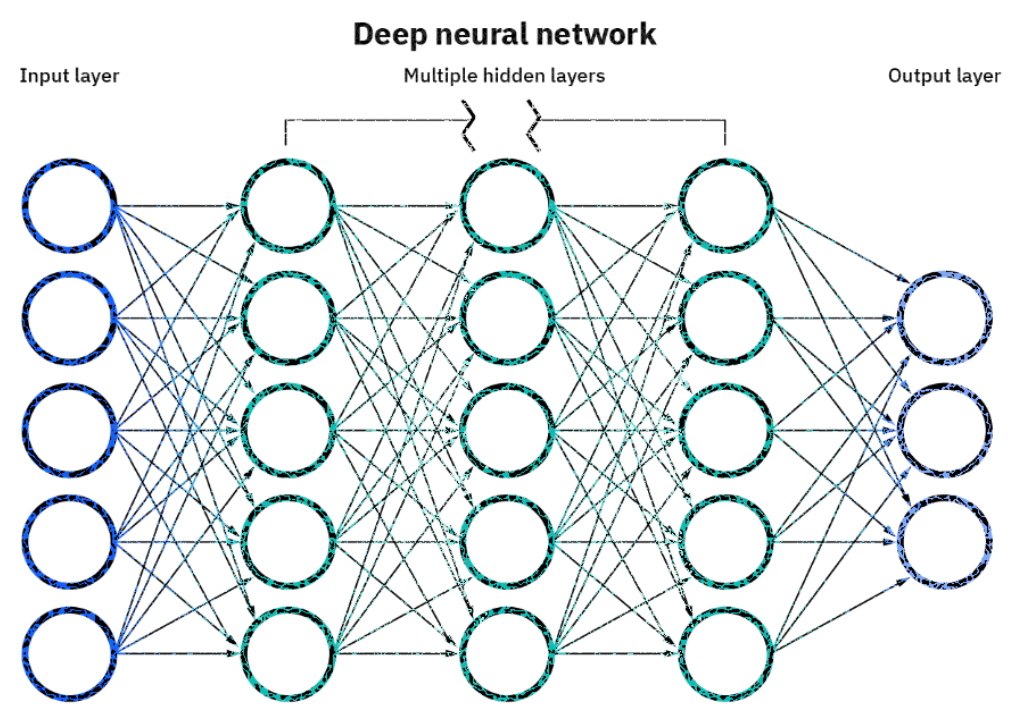

Because diagnosing symptoms of urinary tract infection (UTI) is difficult, (Ozkan et al., 2018) created an ANN-based model to improve UTI diagnostic performance. Using clinically accessible data, this method could distinguish between cystitis and urethritis. Furthermore, they stated that ML approaches can be used to avoid intrusive and costly methods.

It's difficult to tell if a child has had a traumatic brain injury (TBI). As a result, Chong et al., (2015) aimed to investigate the potential of utilizing machine learning to predict moderate to severe TBI. According to their research, the mechanism of head injury as well as clinical information could be used to construct a viable ML model for TBI injury diagnosis. 
Abubakar \& Olatunji, (2020) established a model to forecast the incidence of diarrhea, which is one of the major causes of mortality. The proposed strategy is based on artificial neural networks (ANN) and may be useful in preventing diarrhea. This system has a 95.63 percent accuracy rating.

Prioritizing between severely sick and stable patients in emergency rooms is vital. Raita et al., (2019) created a deep learning (DL) model to predict critical care outcomes. The approach makes use of clinical and demographic data to improve resource usage. When contrasted to the conventional emergency severity index, which is used to prioritize patients, our approach has performed better.

\subsection{Convolutional Neural Network (CNN) in medical diagnosis}

CNN is a sort of feed-forward NN used in artificial intelligence. It's a popular tool for picture recognition. The input data is represented by CNN as multidimensional arrays. It works well when there are a lot of labelled data. The receptive field is what CNN uses to extract each and every piece of the input image. It adds weights to each neuron based on the receptive field's importance. So that it can distinguish between the relevance of neurons.

There are three sorts of layers in CNN's architecture: (1) convolution, (2) pooling, and (3) completely connected.

Internal organs must be visualized in order to discover anomalies in their anatomy or function, which necessitates the use of medical imaging. X-ray, CT, MRI, PET, and ultrasound scanners are medical image capture equipment that capture the structure and function of inside organs and display them as images or movies. For accurate detection of anomalies or diagnosis of functional impairments, the photos and videos must be interpreted. If an anomaly is found, the specific location, size, and shape of the abnormality must be established. Traditionally, trained physicians have done these activities based on their assessment and expertise. These tasks are aimed to be completed by intelligent healthcare systems using CNN and intelligent medical image understanding. The key tasks in medical image interpretation include classification, segmentation, detection, and localization (Le Glaz et al., 2021) (Sarvamangala \& Kulkarni, 2021) (van der Schaar et al., 2021).

\subsection{Medical Diagnostics}

In medical diagnostics, artificial intelligence is commonly used. Al in healthcare aids in the detection of diseases. It also aids in anticipating their progress. Furthermore, with an automated $\mathrm{Al}$ and healthcare screening, address the problem of misdiagnosis (Ishak \& Siraj, 2002). The following are some examples of screening and medical diagnosing solutions: 


\subsection{Al Ultrasound}

An ultrasound is a type of imaging test that employs sound waves to create a picture of organs, tissues, and other structures inside the body (also known as a sonogram). Ultrasounds, unlike x-rays, do not employ radiation (Minoda et al., 2020).

In terms of ease, non-invasiveness, and real-time features, ultrasound imaging outperforms other medical imaging modalities. Computed tomography (CT) exposes patients to radiation, whereas magnetic resonance imaging (MRI) is non-invasive but expensive and timeconsuming (Muse \& Topol, 2020). As a result, US imaging is widely employed in a variety of medical professions for both screening and definitive diagnosis (Kuang et al., 2021).

Deep learning, a popular Al technique, excels in picture pattern identification, making it particularly useful for doctors who rely significantly on images, such as sonologists, radiographers, and pathologists. Despite the fact that obstetric and gynecological ultrasonography are two of the most popular imaging procedures, Al has made minimal impact in this field thus far (Minoda et al., 2020). Nonetheless, Al has a lot of potential for helping with repetitive ultrasound activities like automatically selecting high-quality acquisitions and giving immediate quality assurance. Interdisciplinary interaction between Al developers and ultrasound experts is required for this potential to flourish (Muse \& Topol, 2020).

\subsection{Sleep Diagnostics}

During sleep investigations, billions of biological waveform sample points are recorded, including the ups and downs of the chest as patients breathe, electrical pulses among neurons in dream states, and rapid eye movements (Pépin et al., 2020). These are a few of the signs to understanding a patient's health, but deciphering their meaning and discovering all of the underlying patterns isn't always attainable with the naked eye.

Artificial intelligence (AI) and machine learning could uncover trends in massive datasets of people's health and deliver valuable insights (Shaheen, 2021b). Data collected during sleep and processed by Al could help identify who will get sick in the future, long before any symptoms arise. A restless night's sleep may theoretically indicate who would develop dementia later in life, or variations in heart rate variability could warn the development of pneumonia, data that could be used to follow infectious disease epidemics (Cabrero-Canosa et al., 2004) .

\subsection{Al Cancer Detection Solutions}

In practically every country, cancer is the top cause of mortality and a major impediment to extending life expectancy. Cancer was the primary or second major reason of mortality before the age of 70 in 112 of 183 nations between 2000 and 2019, according to the World Health Organization, and third or fourth in another 23 countries. 
New technologies are the pivot that will allow us to address the healthcare gap in the cancer care continuum. Artificial intelligence (AI) has emerged as a game-changing technology. Clinical treatment driven by Al has the potential to make a significant difference in eliminating health inequities, especially in low-resource settings. Al integration in cancer care can increase diagnosis accuracy and speed, aid healthcare decision, and result in improved health outcomes (Al-shamasneh \& Obaidellah, 2017).

Artificial intelligence (AI) has the potential to improve cancer screening, tumor genetic characterization, drug discovery, and cancer surveillance. With multitudes of genetic and epigenetic changes, cancer is a complicated and multifaceted disease. Al-based algorithms have a lot of potential in terms of detecting genetic abnormalities and abnormal protein interactions early on. Modern biomedical research is also engaged with safely and ethically bringing Al technologies to clinics (Kim et al., 2020).

Despite significant breakthroughs in therapy and diagnosis approaches, cancer is still one of the most lethal diseases. Al may be able to change this in the near future. Its pattern-finding abilities could help physicians strategize treatments and reduce false positives and negatives, potentially leading to cancer cure announcements in the future (Patel et al., 2020).

\subsection{Al Pathology}

The effect of Al will be noticed most strongly in pathology. With more laboratories embracing the shift to digital pathology, the infrastructure needed to deploy these tools will be in place, and their utilization will begin to become a norm in diagnostic practice. In pathology, Al has the potential to develop picture analysis tools that can be utilized for diagnostic assistance or to derive unique understanding into disease biology that are not possible with a human spectator. Some instances of diagnostic support are already available for a small but growing number of applications, including tumor identification, automated tumor grading, immunohistochemistry scoring, and mutation status prediction. There are several obstacles to consider, not the least of which is the verification and regulatory environment for these products (Salto-Tellez et al., 2019) (Tizhoosh \& Pantanowitz, 2018) (Niazi et al., 2019).

\subsection{Al PCR Testing}

The acronym PCR stands for polymerase chain reaction. It's a test that looks for genetic material from a particular creature, like a virus. If we have a virus at the time of the test, the test will detect its existence. Even if we are no longer sick, the test may reveal fragments of the virus (Murphy et al., 2020).

A SARS-CoV-2 virus-specific reverse transcriptase polymerase chain reaction (RT-PCR) test is commonly used to diagnose coronavirus disease 2019 (COVID-19). However, this test can take up to 2 days to complete, serial testing may be necessary to eliminate the possibility of false negative results. Mei et al., (2020) employed artificial intelligence (AI) techniques to combine chest CT images with clinical symptoms, exposure history, and laboratory testing to detect COVID-19 positive individuals quickly in this study. 419 (46.3 percent) of 905 patients tested positive for SARS-CoV-2 using a real-time RT-PCR assay and next-generation sequencing RT- 
PCR. When compared to a senior thoracic radiologist, the Al system attained an area under the curve of 0.92 and had identical sensitivity in a test set of 279 patients. The Al approach also enhanced the detection of COVID-19 positive patients with normal CT scans who were positive by RT-PCR, accurately detecting 17 of 25 (68\%) patients, whereas radiologists categorized all of these patients as COVID-19 negative. The suggested Al system can assist in the quick diagnosis of COVID-19 patients when CT scans and accompanying clinical history are available (Mei et al., 2020).

\subsection{Liver diagnosis}

The liver is the human body's biggest organ. It is in charge of all metabolic operations in the body, from converting nutrients in the diet into useful body chemicals to storing and subsequently providing these substances to the cells as needed. It is also in charge of conversion. transformation of harmful chemicals into non-toxic substances Bile generation is one of the liver's other important activities. protein synthesis, glucose storage and release, haemoglobin processing, blood cleansing, and immunological defence Factor production, bilirubin clearance, and so on. As a result, it is the most important and primary bodily organ. Its health must be maintained in order to improve overall health. However, the reality is that most people In the case of health, it's best to ignore it.

Given the intricacy of the symptoms in the early stages of liver disease, it might be difficult to identify. Because the liver continues to operate even when partially damaged, problems with liver illnesses are often not recognized until it is too late. Early detection has the ability to save lives. The early symptoms of various disorders can be noticed, even if they are not visible to even the most expert medical practitioner. Patients that are diagnosed early in their lives have a much longer life expectancy.

Machine learning algorithms have been developed to predict disease risk and outcomes using multiple clinical parameters, such as assessing liver fibrosis and steatosis, forecasting liver decompensation in primary sclerosing cholangitis, inspection and selecting liver transplant recipients, and predicting post-transplant survival and complications (Decharatanachart et al., 2021).

\subsection{Detects diseases at early stages}

The importance of early detection: delaying medical treatment has the potential to have devastating repercussions. Many doctors and hospitals across the country have seen a major decline in patients seeking everything from basic checks to cancer tests like mammograms, colonoscopies, and even pediatric care.

Delaying care that delays the discovery, diagnosis, and treatment of major health disorders may result in a lower quality of life, illnesses worsening rather than being treated, and even fatalities that may have been avoided. According to preliminary evaluations from hospitals across the country, life-saving treatments including cardiac catheterization to treat a major, 
possibly fatal form of a heart attack and the number of persons diagnosed with cancer are both on the decline (Etzioni et al., 2003).

In many critical sickness cases, the prognosis for treatment is determined by how quickly the diseases are diagnosed. Ideally, symptoms appear early enough for us to recognize that something is incorrect and for us to seek professional assistance. However, certain diseases lack early warning signs, and we frequently hear of cases when early warning signs arrive too late (Jiang et al., 2017).

Furthermore, many people may not consult medical specialists on a regular basis. For some, the time it takes to see a doctor may be a barrier as well. This is where Al applications may assist in performing first-level screens to detect tiny characteristics that may indicate underlying concerns and then referring them to professionals (Shen et al., 2019).

\subsection{Diabetic retinopathy}

Diabetic retinopathy is a disorder that affects eyesight by causing lesions on the retina. DR affects many diabetes people without causing any symptoms in the early stages. If these asymptomatic patients do not seek medical care from an eye specialist as soon as possible, further stages of DR may develop, resulting to irreversible blindness (Padhy et al., 2019). Timely diagnosis and treatment can help prevent $\mathrm{DR}$, which is one of the primary causes of blindness.

By detecting DR problems in their early and asymptomatic stages, Deep Learning can help to reduce some of the issues. This might imply that more diabetes individuals will be screened, and, more crucially, that an early diagnosis of DR will be achievable, allowing the patient to be transferred to an eye expert for treatment. This is feasible thanks to color fundus photos (CFP) (Grzybowski et al., 2020) (Wong \& Bressler, 2016), which are assessed using deep learning to provide a target outcome forecast. The deep learning method is trained on highquality CFPs that have been rated by professionals for DR severity.

The dataset, which Kaggle provides as one of the many public sources, is a key aspect in deep learning model training. Many articles have used a deep learning approach based on Convolution Neural Networks (CNN) with various improvement strategies to improve accuracy. The use of a predictive DR screening system like this could assist governments deal with the overwhelming issue of diabetes patients becoming blind, as well as reducing the cost of dealing with this problem (Grzybowski et al., 2020).

\subsection{Heart conditions}

Individual components (segments) of the ECG waveform can be identified to diagnose heart problems. To properly detect each section of the ECG wave, researchers seek for minor 
patterns and repeated traits (Chen et al., 2021). Coronary Heart Disease, which is a blockage of the veins that irrigate the heart, Heart Arrhythmias, which happen when the electrical impulses that synchronize heartbeats don't operate properly, and other heart problems can be discovered (Abdel-Motaleb \& Akula, 2012). The ECG waveform is a periodic time-series signal made up of $\mathrm{P}$ waves, QRS complexes, and T waves.

The easiest way to assess this signal's mix of periodicity and characteristic qualities is to use the Conv-LSTM deep learning method. The Conv component is essentially a Convolutional Neural Network (CNN) that extracts features. Long Short-Term Memory (LSTM) is a specific variation of a recurrent neural network (dedicated to processing time-series signals) that extracts the time series characteristic waveform and captures the ECG signal's long-term temporal relationships (Djerioui et al., 2020). Deep learning-based screening for cardiac issues can assist individuals with underlying medical conditions seek expert help sooner and avoid serious medical events. In the above use cases including Visual-Recognition, Outlier Detection, Convolutional neural networks, Al plays a critical role.

\section{Conclusion}

Monitoring and data collection devices are common in modern hospitals, with massive information systems collecting and sharing data. Machine learning technique is now well adapted for evaluating medical data, and there is a lot of work being done in medical diagnosis in micro or small diagnostic situations in particular.

When it comes to using machine learning to diagnose diseases, there are a few challenges to consider. First, machine learning does not have the capacity replace a doctor. While machine learning can help predict the likelihood of developing an illness, it cannot take over all of a specialist's responsibilities. For instance, Machine Learning can assist determine if someone has cancer reasonably rapidly and early, but the therapy must still be selected by the doctor. Good data is required for Machine Learning to succeed. It is useless if the data is of poor quality or if no patterns can be detected in it. Similarly, when a model is trained using a set of data, there's a chance that existing patterns won't apply to fresh data.

Doctors and hospitals must employ newly developed models. Upgrading Machine Learning models is not always part of a doctor's job description. It can be tough to find time for a health professional to assist with data and result verification. Using a trained model that no more delivers accurate forecasts can be risky, especially when human health is at stake. It is also hazardous to abandon a tried-and-true paradigm in favor of one which may not succeed.

\section{References}

Abdel-Motaleb, I., \& Akula, R. (2012). Artificial intelligence algorithm for heart disease diagnosis using phonocardiogram signals. 2012 IEEE International Conference on Electro/Information Technology, 1-6. 
Abubakar, I. R., \& Olatunji, S. O. (2020). Computational intelligence-based model for diarrhea prediction using Demographic and Health Survey data. Soft Computing, 24(7), 5357-5366.

Al-shamasneh, A. R. M., \& Obaidellah, U. H. B. (2017). Artificial intelligence techniques for cancer detection and classification: review study. European Scientific Journal, 13(3), 342-370.

Cabrero-Canosa, M., Hernandez-Pereira, E., \& Moret-Bonillo, V. (2004). Intelligent diagnosis of sleep apnea syndrome. IEEE Engineering in Medicine and Biology Magazine, 23(2), 72-81.

Chen, J., Pu, H., \& Wang, D. (2021). Artificial Intelligence Analysis of EEG Amplitude in Intensive Heart Care. Journal of Healthcare Engineering, 2021.

Chong, S.-L., Liu, N., Barbier, S., \& Ong, M. E. H. (2015). Predictive modeling in pediatric traumatic brain injury using machine learning. BMC Medical Research Methodology, 15(1), 1-9.

Decharatanachart, P., Chaiteerakij, R., Tiyarattanachai, T., \& Treeprasertsuk, S. (2021). Application of artificial intelligence in chronic liver diseases: a systematic review and meta-analysis. BMC Gastroenterology, 21(1), 1-16.

Djerioui, M., Brik, Y., Ladjal, M., \& Attallah, B. (2020). Heart Disease prediction using MLP and LSTM models. 2020 International Conference on Electrical Engineering (ICEE), 1-5.

Etzioni, R., Urban, N., Ramsey, S., McIntosh, M., Schwartz, S., Reid, B., Radich, J., Anderson, G., \& Hartwell, L. (2003). The case for early detection. Nature Reviews Cancer, 3(4), 243-252.

Grzybowski, A., Brona, P., Lim, G., Ruamviboonsuk, P., Tan, G. S. W., Abramoff, M., \& Ting, D. S. W. (2020). Artificial intelligence for diabetic retinopathy screening: a review. Eye, 34(3), 451-460.

Hu, J., Perer, A., \& Wang, F. (2015). Data driven analytics for personalized healthcare. Healthcare Information Management Systems: Cases, Strategies, and Solutions: Fourth Edition, 529-554. https://doi.org/10.1007/978-3-319-20765-0_31

Ishak, W. H. W., \& Siraj, F. (2002). Artificial intelligence in medical application: An exploration. Health Informatics Europe Journal, 16.

Jiang, F., Jiang, Y., Zhi, H., Dong, Y., Li, H., Ma, S., Wang, Y., Dong, Q., Shen, H., \& Wang, Y. (2017). Artificial intelligence in healthcare: past, present and future. Stroke and Vascular Neurology, 2(4).

Kim, H.-E., Kim, H. H., Han, B.-K., Kim, K. H., Han, K., Nam, H., Lee, E. H., \& Kim, E.-K. (2020). Changes in cancer detection and false-positive recall in mammography using artificial intelligence: a retrospective, multireader study. The Lancet Digital Health, 2(3), e138e148.

Kollár, A. (2021a). Betting models using Al: A review on ANN, SVM, and Markov Chain.

Kollár, A. (2021b). Predicting the win probability using logistic regression for top four English Premier League teams.

Kuang, M., Hu, H.-T., Li, W., Chen, S.-L., \& Lu, X.-Z. (2021). Articles that use artificial intelligence for ultrasound: A reader's guide. Frontiers in Oncology, 11.

Le Glaz, A., Haralambous, Y., Kim-Dufor, D.-H., Lenca, P., Billot, R., Ryan, T. C., Marsh, J., Devylder, J., Walter, M., \& Berrouiguet, S. (2021). Machine learning and natural language processing in mental health: Systematic review. Journal of Medical Internet Research, 23(5), e15708.

Lee, E. (2021). How do we build trust in machine learning models? Available at SSRN 
3822437.

Mei, X., Lee, H.-C., Diao, K., Huang, M., Lin, B., Liu, C., Xie, Z., Ma, Y., Robson, P. M., \& Chung, M. (2020). Artificial intelligence-enabled rapid diagnosis of patients with COVID-19. Nature Medicine, 26(8), 1224-1228.

Minoda, Y., Ihara, E., Komori, K., Ogino, H., Otsuka, Y., Chinen, T., Tsuda, Y., Ando, K., Yamamoto, H., \& Ogawa, Y. (2020). Efficacy of endoscopic ultrasound with artificial intelligence for the diagnosis of gastrointestinal stromal tumors. Journal of Gastroenterology, 55(12), 1119-1126.

Murphy, K., Smits, H., Knoops, A. J. G., Korst, M. B. J. M., Samson, T., Scholten, E. T., Schalekamp, S., Schaefer-Prokop, C. M., Philipsen, R. H. H. M., \& Meijers, A. (2020). COVID-19 on chest radiographs: a multireader evaluation of an artificial intelligence system. Radiology, 296(3), E166-E172.

Muse, E. D., \& Topol, E. J. (2020). Guiding ultrasound image capture with artificial intelligence. The Lancet, 396(10253), 749.

Niazi, M. K. K., Parwani, A. V, \& Gurcan, M. N. (2019). Digital pathology and artificial intelligence. The Lancet Oncology, 20(5), e253-e261.

Ozkan, I. A., Koklu, M., \& Sert, I. U. (2018). Diagnosis of urinary tract infection based on artificial intelligence methods. Computer Methods and Programs in Biomedicine, 166, 51-59.

Padhy, S. K., Takkar, B., Chawla, R., \& Kumar, A. (2019). Artificial intelligence in diabetic retinopathy: A natural step to the future. Indian Journal of Ophthalmology, 67(7), 1004.

Panesar, A. (2019). Machine learning and Al for healthcare. Springer.

Patel, D., Shah, Y., Thakkar, N., Shah, K., \& Shah, M. (2020). Implementation of artificial intelligence techniques for cancer detection. Augmented Human Research, 5(1), 1-10.

Pépin, J., Bailly, S., \& Tamisier, R. (2020). Big Data in sleep apnoea: Opportunities and challenges. Respirology, 25(5), 486-494.

Raita, Y., Goto, T., Faridi, M. K., Brown, D. F. M., Camargo, C. A., \& Hasegawa, K. (2019). Emergency department triage prediction of clinical outcomes using machine learning models. Critical Care, 23(1), 1-13.

Sadiq, R., Rodriguez, M. J., \& Mian, H. R. (2019). Empirical models to predict disinfection byproducts (DBPs) in drinking water: an updated review.

Salto-Tellez, M., Maxwell, P., \& Hamilton, P. (2019). Artificial intelligence-the third revolution in pathology. Histopathology, 74(3), 372-376.

Sarvamangala, D. R., \& Kulkarni, R. V. (2021). Convolutional neural networks in medical image understanding: a survey. Evolutionary Intelligence, 1-22.

Saxena, G. (2021). Determining success factors of project with supervised learning. www.aetherarcade.com

Shaheen, M. Y. (2021a). Al in Healthcare: medical and socio-economic benefits and challenges.

Shaheen, M. Y. (2021b). Applications of Artificial Intelligence (Al) in healthcare: A review. Shen, J., Zhang, C. J. P., Jiang, B., Chen, J., Song, J., Liu, Z., He, Z., Wong, S. Y., Fang, P.-H., \& Ming, W.-K. (2019). Artificial intelligence versus clinicians in disease diagnosis: systematic review. JMIR Medical Informatics, 7(3), e10010.

Srivastav, B. (2021). The novel Artificial Neural Network assisted models: A review.

Tanveer, M., Richhariya, B., Khan, R. U., Rashid, A. H., Khanna, P., Prasad, M., \& Lin, C. T. (2020). Machine learning techniques for the diagnosis of Alzheimer's disease: A review. ACM Transactions on Multimedia Computing, Communications, and Applications 
(TOMM), 16(1s), 1-35.

Tizhoosh, H. R., \& Pantanowitz, L. (2018). Artificial intelligence and digital pathology: challenges and opportunities. Journal of Pathology Informatics, 9.

van der Schaar, M., Alaa, A. M., Floto, A., Gimson, A., Scholtes, S., Wood, A., McKinney, E., Jarrett, D., Lio, P., \& Ercole, A. (2021). How artificial intelligence and machine learning can help healthcare systems respond to COVID-19. Machine Learning, 110(1), 1-14.

Wong, T. Y., \& Bressler, N. M. (2016). Artificial intelligence with deep learning technology looks into diabetic retinopathy screening. Jama, 316(22), 2366-2367. 